\title{
Oral contraceptives and the risk of gallbladder disease: a comparative safety study
}

\author{
Mahyar Etminan PharmD MSc, Joseph A.C. Delaney PhD, Brian Bressler MD MSc, James M. Brophy MD PhD
}

\begin{abstract}
Background: Recent concerns have been raised about the risk of gallbladder disease associated with the use of drospirenone, a fourthgeneration progestin used in oral contraceptives. We conducted a study to determine the magnitude of this risk compared with other formulations of oral contraceptives.

Methods: We conducted a retrospective cohort study using the IMS LifeLink Health Plan Claims Database. We included women who were using an oral contraceptive containing ethinyl estradiol combined with a progestin during 1997-2009. To be eligible, women had to have been taking the oral contraceptive continuously for at least six months. We computed adjusted rate ratios (RRs) for gallbladder disease using a Cox proportional hazards model. In the primary analysis, gallbladder disease was defined as cholecystectomy; in a secondary analysis, it was defined as hospital admission secondary to gallbladder disease.
\end{abstract}

Results: We included 2721014 women in the cohort, 27087 of whom underwent surgical or laparoscopic cholecystectomy during the follow-up period. Compared with levonorgestrel, an older second-generation progestin, a small, statistically significant increase in the risk of gallbladder disease was associated with desogestrel (adjusted RR 1.05, 95\% confidence interval [CI] 1.01-1.09), drospirenone (adjusted RR 1.20, 95\% Cl 1.16-1.26) and norethindrone (adjusted RR 1.10, 95\% Cl 1.06-1.14). No statistically significant increase in risk was associated with the other formulations of oral contraceptive (ethynodiol diacetate, norgestrel and norgestimate).

Interpretation: In a large cohort of women using oral contraceptives, we found a small, statistically significant increase in the risk of gallbladder disease associated with desogestrel, drospirenone and norethindrone compared with levonorgestrel. However, the small effect sizes compounded with the possibility of residual biases in this observational study make it unlikely that these differences are clinically significant.
Competing interests: None declared.

This article has been peer reviewed.

Correspondence to:

Mahyar Etminan,

mahyar.etminan@vch.ca, metminan@shaw.ca

CMAJ 2011. DOI:10.1503 /cmaj.110161
$\mathrm{O}$ ral contraceptives are the most popular mode of birth control among women and are used by about 100 million wo men worldwide. ${ }^{1}$ Long-term use of these drugs has been associated with a variety of serious adverse events, including deep vein thrombosis, stroke and pulmonary embolism. ${ }^{2}$ In addition, both estrogen and progesterone have been shown to play an important role in the formation of gallstones. ${ }^{3-5}$ However, the relative risk of gallbladder disease associated with different formulations of oral contraceptives, including newer formulations, is unknown.

Recently, there have been concerns expressed in the media about reports of gallbladder disease necessitating cholecystectomy associated with the use of drospirenone, a fourth-generation progestin. ${ }^{6}$ Drospirenone combined with ethinyl estradiol is primarily marketed as Yaz and Yasmin in Canada and the United States and is one of the most prescribed oral contraceptives in North America, with worldwide sales of $\$ 2$ billion in 2009. ${ }^{7}$ The scientific evidence on the risk of gallbladder disease associated with drospirenone consists of only anecdotal or spontaneous reports in databases of adverse drug events.

A possible link between drospirenone and gallbladder disease may lead to cholecystectomy and possible surgical complications. ${ }^{8}$ If there were a substantial risk of gallbladder disease with drospirenone, this might influence its overall risk-benefit ratio and could prompt physicians to prescribe safer alternatives. Given that women using oral contraceptives have been found to be at increased risk of gallbladder disease compared with women not using oral contraceptives, ${ }^{4}$ any excess risk associated with the use of drospirenone merits quantification within the context of a comparative safety study. 


\section{Methods}

\section{Data sources}

We obtained data from the IMS LifeLink Health Plan Claims Database. This database is the largest of its kind in the United States and captures health information on about 78 million residents representing all geographic areas in the country (midwest, 35\%; northeast, $21 \%$; south, $31 \%$; west, $13 \%) .{ }^{9}$ Data are captured longitudinally, with an average enrolment period of two years. ${ }^{9}$ Data fields include demographic characteristics (age, sex, geographic location), prescription drugs (drug name, quantity, day supply), diagnoses (using the International Classification of Diseases, ninth revision [ICD-9]) and hospital admissions (including visits to an emergency department and surgical procedures). The database is subject to routine quality checks to ensure the validity and completeness of the data, and it has been used in numerous pharmacoepidemiologic and health outcome studies. ${ }^{10}$ In addition, because the data come from more than 104 managed care organizations, they go through rigorous quality checks before they are incorporated in the main database. ${ }^{9}$

\section{Study cohort and exposure definition}

We conducted a retrospective cohort study. We included all women who were using an oral contraceptive containing ethinyl estradiol combined with a progestin during 1997-2009. The progestins studied were norethindrone, ethynodiol diacetate, norgestrel, levonorgestrel, norgestimate, desogestrel and drospirenone.

Cohort entry was defined as the index date (baseline), which occurred after 180 days of continuous exposure to a study drug. We excluded women who had lesser amounts of exposure. To avoid misclassification bias, exposed persontime was computed from the index date. This approach allowed for a sustained exposure period without interruptions.

Cohort members were followed to the end of the study period; to the date they switched to another study drug; to discontinuation of a study drug; to the diagnosis of gallbladder disease; or to the termination of health coverage. Because gallbladder disease has a slow onset, we extended follow-up for an additional six months after cohort members had been censored, to observe any new diagnoses of gallbladder disease that may have developed after a study drug was stopped.

\section{Outcome measures}

For the primary analysis, the outcome of gallbladder disease was defined as having had a cholecystectomy. Information on cholecystectomies was ascertained using Current Procedural Terminology (CPT) codes for both surgical and laparoscopic cholecystectomies (CPT codes 47600, 47605, 47610, 47612, 47620, 47562, 47563, 47564, 49310, 56340 and 56341). These procedure codes are used by surgeons to be reimbursed for their services. They have been

Table 1: Characteristics of women included in the study cohort by type of progestin in oral contraceptive used ( $n=2721014)$

\begin{tabular}{|c|c|c|c|c|c|c|c|}
\hline Characteristic & $\begin{array}{l}\text { Desogestrel } \\
n=351322\end{array}$ & $\begin{array}{c}\text { Drospirenone } \\
n=448287\end{array}$ & $\begin{array}{c}\text { ETD } \\
n=53244\end{array}$ & $\begin{array}{l}\text { Levonorgestrel } \\
n=495748\end{array}$ & $\begin{array}{c}\text { Norethindrone } \\
n=546621\end{array}$ & $\begin{array}{c}\text { Norgestimate } \\
n=722667\end{array}$ & $\begin{array}{l}\text { Norgestrel } \\
n=103125\end{array}$ \\
\hline Age, yr, mean (SD) & $28(6.0)$ & $28(6.1)$ & $29(5.9)$ & $29(6.1)$ & $30(5.8)$ & $27(5.6)$ & $29(6.3)$ \\
\hline $\begin{array}{l}\text { Length of follow-up, d, } \\
\text { mean (SD) }\end{array}$ & 363 (479) & 314 (396) & 377 (492) & 357 (471) & $287(431)$ & $338(433)$ & $351(470)$ \\
\hline $\begin{array}{l}\text { No. with gallbladder } \\
\text { disease }\end{array}$ & 3911 & 4974 & 632 & 5201 & 4789 & 6432 & 1148 \\
\hline \multicolumn{8}{|l|}{ Drug use, $\%$} \\
\hline Statin & 0.39 & 0.39 & 0.59 & 0.50 & 0.44 & 0.29 & 0.64 \\
\hline Fibrate & 0.06 & 0.08 & 0.07 & 0.06 & 0.06 & 0.04 & 0.06 \\
\hline \multicolumn{8}{|l|}{ Medical history, \% } \\
\hline Sickle-cell anemia & 0.04 & 0.03 & 0.02 & 0.04 & 0.05 & 0.03 & 0.04 \\
\hline Diabetes mellitus & 3.13 & 3.18 & 3.07 & 3.01 & 3.11 & 2.33 & 3.55 \\
\hline $\begin{array}{l}\text { Inflammatory bowel } \\
\text { disease }\end{array}$ & 0.47 & 0.50 & 0.55 & 0.46 & 0.42 & 0.36 & 0.47 \\
\hline Pancreatitis & 0.35 & 0.35 & 0.36 & 0.36 & 0.33 & 0.26 & 0.40 \\
\hline Smoking & 5.00 & 4.60 & 5.65 & 5.29 & 5.01 & 4.65 & 5.91 \\
\hline Obesity & 0.36 & 0.42 & 0.37 & 0.34 & 0.34 & 0.23 & 0.36 \\
\hline
\end{tabular}


shown to be well correlated with gallbladder disease and have been used as a marker in previous epidemiologic studies. ${ }^{11}$

For the secondary analysis, we considered the outcome as all hospital admissions secondary to gallbladder disease (ICD-9 codes 574 and 575).

\section{Statistical analysis}

We used the Cox proportional hazards model to estimate hazard ratios for gallbladder disease (cholecystectomy or hospital admission secondary to gallbladder disease). We hypothesized that a possible risk of gallbladder disease with use of oral contraceptives would require at least six months of continuous use of these drugs. Thus, exposure was defined at baseline (after 180 days of exposure). To avoid misclassification bias, we excluded participants who either had an event or left the cohort before 180 days of exposure.

Models were adjusted for available covariates to control for confounding; the covariates were age, calendar time, sickle-cell anemia, diabetes mellitus, inflammatory bowel disease, obesity, pancreatitis, smoking and use of statins and fibrates. Levonorgestrel was used as the reference group because it is the most common progestin used in oral contraceptives. ${ }^{2}$

As a sensitivity analysis, we repeated the study to include women who had two years of continuous use of oral contraceptives. We adjusted all analyses for known confounders.

As an alternative analysis, we developed a propensity score model based on the probability of a participant being exposed to drospirenone or another oral contraceptive. We then used this propensity score as an alternate means of adjusting the Cox model.

Proportionality of hazards were examined graphically by means of log-log survival curves, and no meaningful deviations from proportionality were observed after baseline.

As a final sensitivity analysis, we considered a short time window in case the association between oral contraceptives and gallbladder disease was due to acute exposure to the study drug. For this analysis, we defined exposure as the first day of exposure to a study drug, and we followed participants for 180 days (until censoring, the end of the study period or cholecystectomy).

\section{Results}

The cohort included 2721014 women, with 2460094 person-years of follow-up. A total of 27087 women underwent surgical or laparoscopic cholecystectomy. The mean time to cholecystectomy was 330 days (median 166, interquartile range $29-445$ days). The baseline characteristics of the women were comparable across the different types of progestins (Table 1). Except for fibrate use, the covariates used in the multivariable model were associated with an increased risk of gallbladder disease (age, rate ratio [RR] $1.01,95 \%$ confidence interval [CI] 1.01-1.02; reported smoking, RR 2.06, 95\% CI 1.99-2.14; reported obesity, RR 2.63, 95\% CI 2.41-2.87; diabetes, RR 1.67, 95\% CI 1.59-1.74; inflammatory bowel disease, RR 1.26, 95\% CI 1.13-1.40; pancreatitis, RR 9.56, 95\% CI 9.1010.10; sickle-cell anemia, RR 2.20, 95\% CI 1.363.43); statin use, RR 1.19, 95\% CI 1.07-1.33; and fibrate use, RR 1.01, 95\% CI 0.78-1.32).

The adjusted RRs for gallbladder disease in the primary analysis, involving women continuously exposed to a study drug for six months, are shown in Table 2 and Figure 1. Compared with the use of levonorgestrel, a small, statistically significant increase in the risk of gallbladder disease

\begin{tabular}{|c|c|c|c|}
\hline Progestin & $\begin{array}{l}\text { Crude rate ratio } \\
(95 \% \mathrm{Cl})\end{array}$ & $\begin{array}{l}\text { Adjusted rate ratiot } \\
\qquad(95 \% \mathrm{Cl})\end{array}$ & $\begin{array}{l}\text { Adjusted for } \\
\text { propensity score }\end{array}$ \\
\hline Levonorgestrel & 1.00 (ref) & 1.00 (ref) & 1.00 (ref) \\
\hline Desogestrel & $1.03(0.99-1.08)$ & $1.05(1.01-1.09)$ & $1.03(0.98-1.07)$ \\
\hline Drospirenone & $1.39(1.34-1.44)$ & $1.20(1.16-1.26)$ & $1.13(1.09-1.18)$ \\
\hline Ethynodiol diacetate & $1.03(0.95-1.12)$ & $1.08(0.99-1.25)$ & $1.08(0.99-1.17)$ \\
\hline Norethindrone & $1.12(1.07-1.19)$ & $1.10(1.06-1.14)$ & $1.10(1.06-1.15)$ \\
\hline Norgestimate & $0.97(0.93-1.00)$ & $1.00(0.96-1.04)$ & $0.92(0.89-0.95)$ \\
\hline Norgestrel & 1.07 (1.01-1.15) & $1.06(0.99-1.12)$ & $1.07(1.01-1.48)$ \\
\hline
\end{tabular}


was associated with the use of desogestrel, drospirenone and norethindrone.

A total of 567447 women continuously took an oral contraceptive for two years, with 792871 person-years of follow-up. The mean time to cholecystectomy in this group was 510 days. In this sensitivity analysis, a small, statistically significant increase in the risk of gallbladder disease was associated with the use of drospirenone and ethynodiol diacetate (Table 3).

The association between drospirenone use and gallbladder disease was reduced in both the adjusted and propensity score models, with a number needed to harm of 550 compared with levonorgestrel. There was no clinically meaningful difference in the risk of gallbladder disease with different oral contraceptives in the propensity score analysis (Tables 2 and 3 ).

The results were also consistent in the secondary analysis, in which the outcome was defined as hospital admission secondary to gallbladder disease (Table 4).

A one-sided test of interaction did not show interactions between participants' age and oral contraceptive type.

Finally, the adjusted RRs in the sensitivity analysis for acute exposure showed no association between type of oral contraceptive and gallbladder disease aside from drospirenone, for which a protective effect was observed (desogestrel, RR 0.93, 95\% CI 0.86-1.00; drospirenone, RR $0.87,95 \%$ CI 0.81-0.93; ethynodiol diacetate, RR 0.92 , 95\% CI $0.78-1.01$; norethindrone, RR 1.10, 95\% CI 1.04-1.18; norgestimate, RR 0.78 , 95\% CI 0.73-0.83; and norgestrel, RR 0.95, 95\% CI 0.87-1.07).

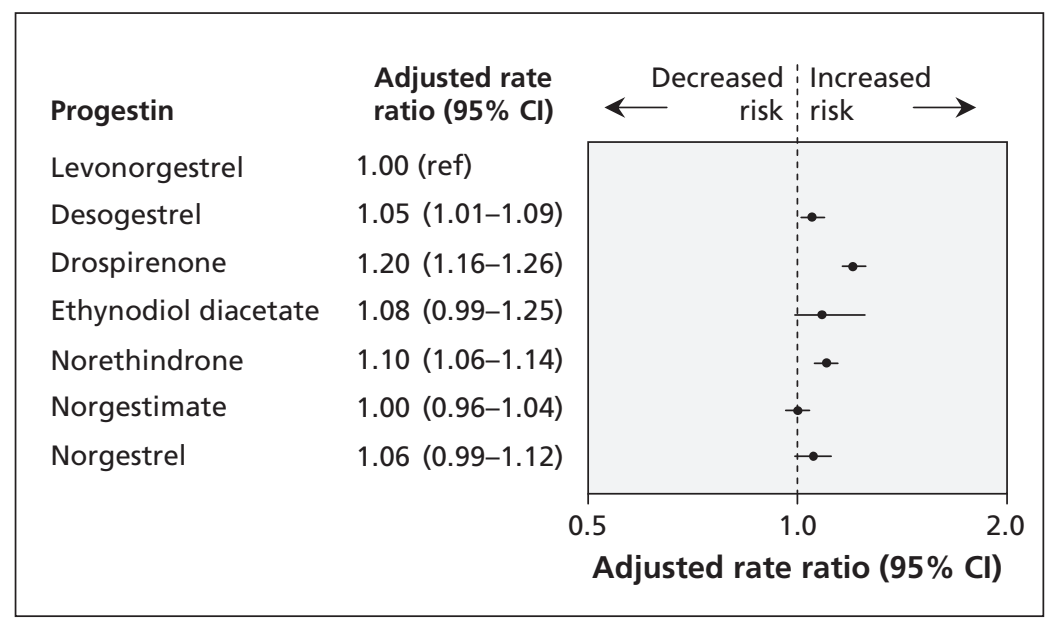

Figure 1: Risk of gallbladder disease (as defined by occurrence of cholecystectomy) associated with six months of continuous use of oral contraceptives containing different progestins. Women using levonorgestrel served as the reference group. Rate ratios were adjusted for age, calendar time, sickle-cell anemia, diabetes mellitus, inflammatory bowel disease, obesity, pancreatitis, smoking, and use of statins and fibrates. A rate ratio greater than 1.0 indicates an increased risk of gallbladder disease. $\mathrm{Cl}$ = confidence interval, ref = reference group.

\section{Interpretation}

In this large cohort of women using oral contraceptives, we found a small, statistically significant increase in the risk of gallbladder disease associated with desogestrel, drospirenone and norethindrone compared with levonorgestrel. However, this difference is unlikely to be clinically important. Moreover, given the observational nature of this study and the fact that adjusting for covariates leads to estimates being closer to the null suggests that residual confounding may explain, at least in part, these small differences.

Studies have shown that long-term use of an oral contraceptive is associated with an increased risk of gallbladder disease compared with no use. A cohort study using data from the Nurse's Health Study found a slight increase in the risk of gallstones among women who had used oral contraceptives for 15 years or longer (RR 1.5, 95\% CI 1.10-2.20). ${ }^{5}$ Similarly, a meta-analysis of 26 observational studies found a $36 \%$ increase in the development of gallbladder disease among women who were using oral contraceptives compared with those not taking these drugs. ${ }^{4}$ Both estrogen and progesterone have been shown to increase the risk of gallstones. ${ }^{12}$ Estrogen has been shown to increase cholesterol production in the liver, with excess amounts precipitating in bile and leading to the formation of gallstones. ${ }^{13}$ Progesterone has been shown to decrease gallbladder motility, which impedes bile flow and leads to gallstone formation..$^{14}$

In our study, there was a high discontinuation rate in the primary cohort, such that only $20 \%$ of the original cohort was continuously exposed to an oral contraceptive for two years. Given the likelihood of adverse events and availability of a wide range of oral contraceptives, women are increasingly unlikely to continue with only one type of oral contraceptive..$^{15}$ In one study, up to $46 \%$ of women who started taking an oral contraceptive stopped the drug after six months. ${ }^{15}$

Our data do not show that the increased risk of gallbladder disease associated with drospirenone is clinically meaningful compared with other formulations of oral contraceptives. Drospirenone had worldwide sales of $\$ 2$ billion in 2009, making it one of the most prescribed oral contraceptives in North America. ${ }^{7}$ The surge in the number of reported cases of gallbladder disease facilitated through the media may have contributed in making drospirenone appear to be associated with a higher risk of gallbladder disease compared with older contraceptives. 


\section{Strengths and limitations}

Use of the IMS LifeLink Health Plan Claims Database allowed us to examine the risk of gallbladder disease among close to 2.5 million women continuously exposed to different formulations of oral contraceptives, including the newer drospirenone. This attribute of the database, crucial in a study comparing the safety of oral contraceptives, is difficult or impossible to achieve with other equally valid databases owing to sample size limitations. In addition, the in crease in the risk of gallbladder disease associated with covariates such as reported smoking and obesity is consistent with findings in the literature and adds face validity to our study. ${ }^{16}$

We also took several steps to control for confounding bias that may threaten the validity of pharmacoepidemiologic cohort studies. ${ }^{17} \mathrm{By}$ design, this study was restricted to all women using oral contraceptives, allowing study participants to share similar characteristics (Table 1). This type of restriction has been used to reduce the risk of confounding by indication in pharmacoepidemiologic studies. ${ }^{18}$ The use of an active comparator will ensure that any confounding by indication or contraindication is minimized. We also conducted sensitivity analyses in which we examined the risk of gallbladder disease with different exposure periods. The slight protective effect observed with only one prescription of drospirenone may have been due to random error or possible channeling bias, including the possibility that clinicians may have been less likely to prescribe drospirenone to women who may have been more prone to gallbladder disease.

Immortal time bias is another bias that has been noted in various pharmacoepidemiologic studies. ${ }^{19}$ This is a bias whereby users of a drug may have a specious survival advantage over nonusers by study design owing to misclassification of exposure time, which makes the intervention seem protective. ${ }^{18,19}$ In our study, misclassification was avoided by defining exposure to six months of continuous use as well as computing exposed person-time at the index date.

The prescribing of oral contraceptives may be influenced by heavy marketing from manufacturers. By controlling for calendar time, we were able to control for secular trends in prescribing of oral contraceptives that may usually favour the prescribing of one oral contraceptive over another.

As with all pharmacoepidemiologic studies that use claims data, our study has limitations. The ICD-9 codes for gallbladder disease in most administrative databases, including the IMS LifeLink Health Plan Claims Database, have not been validated. This is primarily the reason why we used CPT (Common Procedures and Terminology) codes for the primary analysis; CPT codes have been shown to be well correlated with gallbladder disease. ${ }^{11}$

Body mass index and ethnicity are two variables that we could not control for in this study. Body mass index is a possible confounder because drospirenone was marketed for having the least effect on weight, which may have prompted clinicians to prescribe it to heavier women. Such a bias, if present, would have made drospirenone appear more harmful with respect to gallbladder disease.

Residual confounding with other known and unknown variables may also have affected our results. For example, our data lacked information on diet, which is a potential confounder in this study. Also, we only had data on reported smoking and obesity.

Despite these limitations, given the small magnitude of the relative risks in this large co-

Table 3: Risk of gallbladder disease* associated with two years of continuous use of oral contraceptives, by type of progestin

\begin{tabular}{|llll|}
\hline Progestin & $\begin{array}{c}\text { Crude rate } \\
\text { ratio }(95 \% \mathrm{Cl})\end{array}$ & $\begin{array}{c}\text { Adjusted rate } \\
\text { ratiot }(95 \% \mathrm{Cl})\end{array}$ & $\begin{array}{c}\text { Adjusted for } \\
\text { propensity score }\end{array}$ \\
\hline Levonorgestrel & $1.00($ ref $)$ & $1.00($ ref $)$ & $1.00($ ref $)$ \\
\hline Desogestrel & $0.99(0.94-1.04)$ & $1.02(0.96-1.07)$ & $0.99(0.93-1.04)$ \\
\hline Drospirenone & $1.50(1.45-1.60)$ & $1.30(1.23-1.37)$ & $1.19(1.14-1.26)$ \\
\hline Ethynodiol diacetate & $1.11(1.00-1.23)$ & $1.17(1.06-1.30)$ & $1.17(1.06-1.30)$ \\
\hline Norethindrone & $1.02(0.97-1.07)$ & $1.04(0.99-1.10)$ & $1.06(1.00-1.11)$ \\
\hline Norgestimate & $0.95(0.91-0.99)$ & $0.98(0.93-1.03)$ & $0.87(0.83-0.91)$ \\
\hline Norgestrel & $1.09(1.00-1.18)$ & $1.06(0.98-1.15)$ & $1.10(1.01-1.91)$ \\
\hline
\end{tabular}

Note: $\mathrm{Cl}=$ confidence interval, ref = reference group.

*Patients with gallbladder disease were defined as those who underwent cholecystectomy. †Adjusted for age, calendar time, sickle-cell anemia, diabetes mellitus, inflammatory bowel disease, obesity, pancreatitis, smoking, and use of statins and fibrates.

Table 4: Risk of hospital admission secondary to gallbladder disease associated with six months of continuous use of oral contraceptives, by type of progestin

\begin{tabular}{|llc|}
\hline Progestin & $\begin{array}{c}\text { Crude rate ratio } \\
(95 \% \mathrm{Cl})\end{array}$ & $\begin{array}{c}\text { Adjusted rate ratio* } \\
(95 \% \mathrm{Cl})\end{array}$ \\
\hline Levonorgestrel & $1.00($ ref $)$ & 1.00 (ref) \\
\hline Desogestrel & $0.95(0.94-0.97)$ & $0.99(0.98-1.00)$ \\
\hline Drospirenone & $1.45(1.43-1.46)$ & $1.10(1.09-1.12)$ \\
\hline Ethynodiol diacetate & $0.95(0.93-0.97)$ & $1.04(1.01-1.06)$ \\
\hline Norethindrone & $1.07(1.06-1.08)$ & $1.09(1.08-1.10)$ \\
\hline Norgestimate & $1.00(0.99-1.01)$ & $0.99(0.98-1.00)$ \\
\hline Norgestrel & $1.08(1.06-1.10)$ & $1.06(1.05-1.08)$ \\
\hline
\end{tabular}

Note: $\mathrm{Cl}=$ confidence interval, ref = reference group.

*Adjusted for age, calendar time, sickle-cell anemia, diabetes mellitus, inflammatory bowel disease, obesity, pancreatitis, smoking, and use of statins and fibrates. 
hort study, it would require a large and prevalent confounder to alter the interpretation of the study data.

\section{Conclusion}

In a large cohort of women using oral contraceptives, we found a small, statistically significant increase in the risk of gallbladder disease associated with the use of desogestrel, drospirenone and norethindrone compared with levonorgestrel. However, the small effect sizes compounded with the possibility of residual biases in this observational study make it unlikely that these differences are clinically significant.

\section{References}

1. The World Health Organization. Cardiovascular disease and steroid hormone contraception. Technical Report Series 877. Geneva: The Organization; 1998.

2. van Hylckama Vlieg A, Helmerhorst FM, Vandenbroucke JP, et al. Effects of oestrogen dose and progestogen type on venous thrombotic risk associated with oral contraceptives: results of the MEGA case-control study. BMJ 2009;339:b2921.

3. Layde PM, Vessey MP, Yeates D. Risk factors for gall-bladder disease: a cohort study of young women attending family planning clinics. J Epidemiol Community Health 1982;36:274-8.

4. Thijs C, Knipschild P. Oral contraceptives and the risk of gallbladder disease: a meta-analysis. Am J Public Health 1993;83:1113-20.

5. Grodstein F, Colditz GA, Hunter DJ, et al. A prospective study of symptomatic gallstones in women: relation with oral contraceptives and other risk factors. Obstet Gynecol 1994;84:207-14.

6. CTV News Yasmin/Yaz birth control pill users launch lawsuit. Scarborough (ON): CTV Inc.; 2010. Available: www.ctv.ca /CTVNews/EdmontonHome/20100310/birth_control_100310 (accessed 2010 Dec. 24).

7. Bayer annual report, 2009. Leverkusen (Germany): Bayer; 2010. Available: www.annualreport2009.bayer.com/en/BayerAnnual-Report-2009.pdfx (accessed 2010 Dec. 24).

8. Fletcher DR, Hobbs MS, Tan P, et al. Complications of cholecystectomy: risks of the laparoscopic approach and protective effects of operative cholangiography: a population-based study. Ann Surg 1999;229:449-57.

9. IMS Health. LifeLink health plan claims database: overview and study design issues. The American Medical Association; 2010. Available: http://uams.edu/cctr/hsrcore/Lifelink_Health _Plan_Claims_Data_DesignIssues_wcost_April2010\%5B1\%5D pdf (accessed 2011 Feb. 29)

10. Forrest CB, Lemke KW, Bodycombe DP, et al. Medication, diag- nostic, and cost information as predictors of high-risk patients in need of care management. Am J Manag Care 2009;15:41-8.

11. Simon JA, Hunninghake DB, Agarwal SK, et al. Effect of estrogen plus progestin on risk for biliary tract surgery in postmenopausal women with coronary artery disease: the Heart and Estrogen/Progestin Replacement Study. Ann Intern Med 2001;135:493-501.

12. George ED, Schluger LK. Special women's health issues in hepatobiliary diseases. Clin Fam Pract 2000;2:155-69.

13. Donovan JM. Physical and metabolic factors in gallstone pathogenesis. Gastroenterol Clin North Am 1999;28:75-97.

14. George ED, Schluger LK. Special women's health issues in hepatobiliary diseases. Clin Fam Pract 2000;2:155-69.

15. Rosenberg MJ, Waugh MS. Oral contraceptive discontinuation: a prospective evaluation of frequency and reasons. Am J Obstet Gynecol 1998;179:577-82.

16. Strom BL, Tamragouri RN, Morse ML, et al. Oral contraceptives and other risk factors for gallbladder disease. Clin Pharmacol Ther 1986;39:335-41.

17. Suissa S, Garbe E. Primer: administrative health databases in observational studies of drug effects - advantages and disadvantages. Nat Clin Pract Rheumatol 2007;3:725-32.

18. Psaty BM, Siscovick DS. Minimizing bias due to confounding by indication in comparative effectiveness research: the importance of restriction. JAMA 2010;304:897-8.

19. Suissa S. Immortal time bias in pharmaco-epidemiology. Am J Epidemiol 2008;15:492-9.

Affiliations: From the Department of Medicine (Etminan, Bressler), Faculty of Medicine, University of British Columbia, Vancouver, BC; the Centre for Clinical Epidemiology and Evaluation (Etminan), Vancouver Coastal Health Institute, Vancouver, BC; the College of Pharmacy (Delaney), University of Florida, Gainesville, Fla.; and the Department of Medicine (Brophy), Faculty of Medicine, McGill University, Montréal, Que.

Contributors: Mahyar Etminan, Joseph Delaney and James Brophy contributed to the study concept and design, the acquisition of data and the drafting of the manuscript. All of the authors contributed to the analysis and interpretation of the data and the critical revision of the manuscript for important intellectual content. All of the authors approved the final version of the manuscript submitted for publication. Joseph Delaney was responsible for the statistical analysis and is the study guarantor.

Funding: The study was funded by an operating grant provided in part by the Fonds de la recherche en santé du Québec (FRSQ), the Ministère de la Santé et des Services sociaux and the McGill University Health Center. James Brophy is the recipient of a career award from the FRSQ.

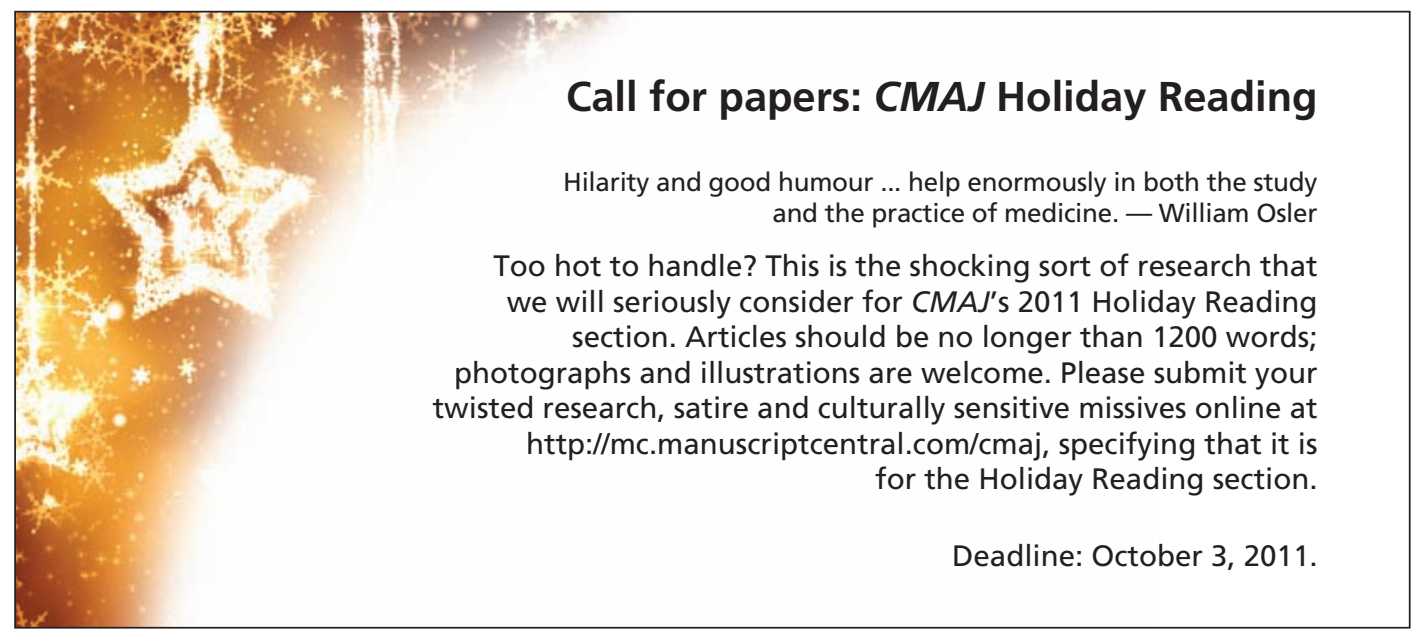

